\title{
A CHARACTERIZATION OF THE SYMMETRICALLY BALANCED VCG RULE IN THE QUEUEING PROBLEM
}

\author{
YOUNGSUB CHUN, MANIPUSHPAK MITRA, AND SURESH MUTUSWAMI
}

\begin{abstract}
We characterize the symmetrically balanced VCG rule in the queueing problem using the axioms of outcome efficiency, budget balance, equal treatment of equals, Pareto indifference, together with a weakening of strategy-proofness, upward-invariance.
\end{abstract}

JEL Classifications: C72, D63, D82.

Keywords: Queueing problem, symmetrically balanced VCG rule, axiomatic characterization, simple proof.

\section{INTRODUCTION}

A group of agents must be served in a facility. The facility serves only one agent at a time and agents incur idiosyncratic waiting costs. Waiting costs are linear in time and an agent's waiting cost is known only to the agent. An agent's utility is the difference between the monetary transfer received and her waiting costs. The objective is to determine the order in which to serve agents and the monetary transfers they should receive. This queueing problem has been analyzed extensively from various perspectives (Dolan [3], Suijs [10], Mitra [8], Maniquet [7], Chun [1], [2], Mitra and Mutuswami [9], and others) and many allocation rules have been proposed.

The symmetrically balanced VCG rule has many nice properties. It is strategyproof, outcome efficient and budget balanced and hence "first-best" implementable (Mitra [8], Suijs [10]). It is also envy-free

Date: March 23, 2015.

We thank Çağatay Kayi, Eve Ramaekers, William Thomson, the referees and the associate editor for their comments. Chun's work was supported by the National Research Foundation of Korea Grant funded by the Korean Government (NRF2013S1A3A2055391) and the Center for Distributive Justice, Seoul National University.

Department of Economics, Seoul National University, Seoul, South Korea. e-mail: ychun@snu.ac.kr.

Economic Research Unit, Indian Statistical Institute, Kolkata-700035, India. e-mail: mmitra@isical.ac.in.

Department of Economics, University of Leicester, Leicester LE1 7RH, United Kingdom. e-mail: sm403@le.ac.uk. 
(Chun [2]). Allocation rules having good strategic and fairness properties are rare and indicates the importance of this rule.

Recently, Kayi and Ramaekers [5] provided an axiomatic characterization of the symmetrically balanced VCG rule (which they call the largest equally distributed pairwise pivotal rule). We show, however, through a counter-example that their axioms are not sufficient to characterize the rule (for details, see Remark 3.3). In a corrigendum, Kayi and Ramaekers [6] provide a corrected statement of their characterization. Here, we present another characterization of the rule using the axioms of outcome efficiency, budget balance, equal treatment of equals, Pareto indifference, together with a weakening of strategyproofness, upward-invariance. The axioms are similar to that used by Kayi and Ramaekers [5] but our proof is very simple.

\section{PRELIMINARIES}

2.1. The queueing problem. Let $N=\{1, \ldots, n\}, n \geq 3$, be the set of agents. ${ }^{1}$ Each agent has one job to process but only one job can be processed at a time. All jobs take the same time to process which is normalized to one.

A queue is an onto function $\sigma: N \rightarrow\{1, \ldots, n\}$ denoting the order in which jobs are processed. We denote $\sigma(i)$ as $\sigma_{i}$. The set of predecessors of agent $i$ is $P_{i}(\sigma)=\left\{j \in N \mid \sigma_{j}<\sigma_{i}\right\}$ and the set of followers is $F_{i}(\sigma)=\left\{j \in N \mid \sigma_{j}>\sigma_{i}\right\}$. The set of all possible queues is denoted $\Sigma(N)$.

Agent $i$ 's waiting cost per unit of time is denoted $\theta_{i} \in \mathbb{R}_{+}$and is known only to her. A profile, $\theta=\left(\theta_{i}\right)_{i \in N}$, is a collection of the waiting costs of all agents. For all $i \in N, \theta_{N \backslash\{i\}}$ denotes the collection of waiting costs of all agents other than $i$. An allocation for $\theta$ is a pair $(\sigma, t)$, where $\sigma \in \Sigma(N)$ is the chosen queue and $t=\left(t_{1}, \ldots, t_{n}\right)$ is the set of monetary transfers to the agents. An allocation is feasible if the sum of transfers is not positive. The set of all feasible allocations for $\theta$ is $Z(\theta)=\left\{(\sigma, t) \in \Sigma(N) \times \mathbb{R}^{n} \mid \sum_{i=1}^{n} t_{i} \leq 0\right\}$. Agents have quasilinear preferences and an agent's utility is given by $u_{i}\left(\sigma_{i}, t_{i} ; \theta_{i}\right)=$ $-\left(\sigma_{i}-1\right) \theta_{i}+t_{i}$.

An allocation rule, or simply a rule, is a mapping $\varphi$ which associates to each profile $\theta$, a non-empty subset $\varphi(\theta)$ of feasible allocations. For all $(\sigma, t) \in \varphi(\theta)$, let $u_{i}\left(\sigma_{i}, t_{i} ; \theta_{i}^{\prime}\right)=-\left(\sigma_{i}-1\right) \theta_{i}^{\prime}+t_{i}$ denote $i$ 's utility in $\varphi$ when the announced profile is $\theta$ and her own waiting $\cos t$ is $\theta_{i}^{\prime}$.

\footnotetext{
${ }^{1}$ We exclude $n=2$ because the symmetrically balanced VCG rule, which is the focus of our analysis is defined only for $n \geq 3$.
} 
In what follows, vector inequalities are denoted $<, \leq$, and $\leqq$. $^{2}$

2.2. Axioms. A queue $\sigma$ is efficient for the profile $\theta$ if $\sigma \in$ $\operatorname{argmin}_{\sigma^{\prime} \in \Sigma(N)} \sum_{i \in N}\left(\sigma_{i}^{\prime}-1\right) \theta_{i}$. It is easy to see that efficiency implies $\sigma_{i}<\sigma_{j}$ whenever $\theta_{i}>\theta_{j}$. For all profiles $\theta$, let $E(\theta)$ be the set of all efficient queues. Note that $E(\theta)$ is always non-empty and is a singleton if no two agents have the same waiting cost.

Now we are ready to introduce our axioms. Queue efficiency requires that a rule should choose efficient queues. Budget balance requires that the sum of transfers by a rule should be equal to zero. Equal treatment of equals requires that two agents with the same waiting cost should end up with the same utilities. Pareto indifference requires that for all profiles, if an allocation is chosen by a rule and there is another feasible allocation which gives the same utility to each agent, then this alternative should also be chosen by the rule. Our final axiom is upward-invariance which requires that an agent's transfer not be affected by misrepresenting her waiting cost upward if the queue is unaffected.

Definition. A rule $\varphi$ is queue efficient (EFF) if for all $\theta$ and all $(\sigma, t) \in$ $\varphi(\theta), \sigma \in E(\theta)$.

Definition. A rule $\varphi$ is budget balanced (BB) if for all $\theta$ and all $(\sigma, t) \in$ $\varphi(\theta), \sum_{i=1}^{n} t_{i}=0$.

Remark 2.1. Since $u_{i}\left(\sigma_{i}, t_{i} ; \theta_{i}\right)=-\left(\sigma_{i}-1\right) \theta_{i}+t_{i}$, BB can alternatively be written as $\sum_{i \in N} u_{i}\left(\sigma_{i}, t_{i} ; \theta_{i}\right)=-\sum_{i \in N}\left(\sigma_{i}-1\right) \theta_{i}$. We use this observation throughout the proof of the main result, Theorem 3.1.

Definition. A rule $\varphi$ satisfies equal treatment of equals (ETE) if for all $\theta$, all $i, j \in N$, and all $(\sigma, t) \in \varphi(\theta)$, if $\theta_{i}=\theta_{j}$, then $u_{i}\left(\sigma_{i}, t_{i} ; \theta_{i}\right)=$ $u_{j}\left(\sigma_{j}, t_{j} ; \theta_{j}\right)$.

Definition. A rule $\varphi$ satisfies Pareto indifference (PI) if for all $\theta$, all $(\sigma, t) \in \varphi(\theta)$ and all $\left(\sigma^{\prime}, t^{\prime}\right) \in Z(\theta)$ such that $u_{i}\left(\sigma_{i}^{\prime}, t_{i}^{\prime} ; \theta_{i}\right)=$ $u_{i}\left(\sigma_{i}, t_{i} ; \theta_{i}\right)$ for all $i \in N,\left(\sigma^{\prime}, t^{\prime}\right) \in \varphi(\theta)$.

Call $\theta$ and $\theta^{\prime} i$-variants if $\theta_{j}=\theta_{j}^{\prime}$ for all $j \in N \backslash\{i\}$.

Definition. A rule $\varphi$ satisfies upward-invariance (U-INV) if for all $i \in$ $N$, all $i$-variants $\theta, \theta^{\prime}$ such that $\theta_{i}^{\prime}>\theta_{i}$, all $(\sigma, t) \in \varphi(\theta)$, and all $\left(\sigma^{\prime}, t^{\prime}\right) \in \varphi\left(\theta^{\prime}\right)$, if $\sigma\left(\theta^{\prime}\right)=\sigma(\theta)$, then $t_{i}\left(\theta^{\prime}\right)=t_{i}(\theta)$.

Remark 2.2. It is easy to prove that there are no rules satisfying EFF, BB, ETE and U-INV when $n=2$.

${ }^{2} x<y$ if $x_{i}<y_{i}$ for all $i ; x \leq y$ if $x_{i} \leq y_{i}$ for all $i$ with at least one strict inequality; $x \leqq y$ if $x_{i} \leq y_{i}$ for all $i$. 
Kayi and Ramaekers [5] do not use U-INV; instead they use two variants of strategy-proofness. We now define these axioms to facilitate a comparison.

Definition. Let $\theta$ and $\theta^{\prime}$ be $i$-variants. A mechanism is strongly strategy-proof (SP) if for all $i \in N$, all $(\sigma, t) \in \varphi(\theta)$, and all $\left(\sigma^{\prime}, t^{\prime}\right) \in \varphi\left(\theta^{\prime}\right), u_{i}\left(\sigma_{i}, t_{i} ; \theta_{i}\right) \geq u_{i}\left(\sigma_{i}^{\prime}, t_{i}^{\prime} ; \theta_{i}\right)$. It is weakly strategyproof (WSP) if for all $i \in N$, all $(\sigma, t) \in \varphi(\theta)$, and all $\left(\sigma^{\prime}, t^{\prime}\right) \in$ $\varphi\left(\theta^{\prime}\right)$, (i) $\max _{(\sigma, t) \in \varphi(\theta)} u_{i}\left(\sigma, t ; \theta_{i}\right) \geq \max _{\left(\sigma^{\prime}, t^{\prime}\right) \in \varphi\left(\theta^{\prime}\right)} u_{i}\left(\sigma^{\prime}, t^{\prime} ; \theta_{i}\right)$ and (ii) $\min _{(\sigma, t) \in \varphi(\theta)} u_{i}\left(\sigma, t ; \theta_{i}\right) \geq \min _{\left(\sigma^{\prime}, t^{\prime}\right) \in \varphi\left(\theta^{\prime}\right)} u_{i}\left(\sigma^{\prime}, t^{\prime} ; \theta_{i}\right)$

Remark 2.3. Clearly, while SP and WSP imply U-INV, the reverse implication does not hold: the mechanism in Remark 3.5(1) is an example of a mechanism which satisfies U-INV but neither SP nor WSP. Note that in this mechanism, an increase in an agent's waiting cost can result in her being assigned an inferior queue position. This is incompatible with SP and WSP but compatible with U-INV.

\section{THE SYMMETRICALLY BALANCED VCG RULE}

The symmetrically balanced VCG rule (henceforth, the SB rule) $\varphi^{S B}$, defined for $n \geq 3$, is the rule such that for all profiles $\theta$,

$$
\begin{aligned}
& \varphi^{S B}(\theta)=\left\{\left(\sigma^{S B}, t^{S B}\right) \in Z(\theta) \mid \sigma^{S B} \in E(\theta) \text { and } \forall i \in N\right. \\
&\left.t_{i}^{S B}=\sum_{\ell \in P_{i}\left(\sigma^{S B}\right)}\left(\frac{\sigma_{\ell}^{S B}-1}{n-2}\right) \theta_{\ell}-\sum_{\ell \in F_{i}\left(\sigma^{S B}\right)}\left(\frac{n-\sigma_{\ell}^{S B}}{n-2}\right) \theta_{\ell}\right\} .
\end{aligned}
$$

Remark 3.1. The SB rule satisfies BB no matter which efficient queue is chosen. From the expression for $t_{i}^{S B}$, it follows that the rule is essentially single-valued in the sense that each agent's utility is the same in all efficient queues. Moreover, all efficient queues and the corresponding transfers are chosen by the SB rule.

The following theorem is the main result of the paper.

Theorem 3.1. Let $n \geq 3$. A rule satisfies EFF, BB, ETE, PI, and U-INV if and only if it is the SB rule.

Proof. Kayi and Ramaekers [5] show that the SB rule satisfies EFF, BB, ETE, and SP. By Remark 2.3, it satisfies U-INV. By Remark 3.1, it also satisfies PI. We now prove the converse. Let $\varphi$ be a rule satisfying 
the five axioms. By Remark 3.1, it suffices to show that at all profiles $\theta$, each agent's utility is the same as her utility in the SB rule. ${ }^{3}$

Let $\theta^{\prime}=\left(\theta_{i}^{\prime}\right)_{i \in N}$ be an arbitrary profile. Since we can always rename agents, we assume without loss of generality that $\theta_{1}^{\prime} \geq \cdots \geq$ $\theta_{n}^{\prime}$. Let $\Theta$ be the set of profiles $\theta$ satisfying
A1. $\theta_{i} \in\left\{\theta_{1}^{\prime}, \ldots, \theta_{n}^{\prime}\right\}, i=1, \ldots, n$,
A2. $\theta_{i+1} \leq \theta_{i} \leq \theta_{i}^{\prime}, i=1, \ldots, n-1$,
A3. $\theta_{n}=\theta_{n}^{\prime}$.

Note that the set $\Theta$ associated with $\theta^{\prime}$ can be obtained by sequentially and recursively replacing $\theta_{i}^{\prime}$ by $\theta_{i+1}^{\prime}$ if $\theta_{i}^{\prime} \neq \theta_{i+1}^{\prime}$. The sequence of changes for $n=4$ is given in Figure 1. The smallest element of $\Theta$ is $\left(\theta_{n}^{\prime}, \ldots, \theta_{n}^{\prime}\right)$ and the largest element $\theta^{\prime}=\left(\theta_{1}^{\prime}, \ldots, \theta_{n}^{\prime}\right)$.

By (A2), for all $\theta \in \Theta, \theta_{1} \geq \cdots \geq \theta_{n}$. Let $(\sigma, t) \in \varphi(\theta)$. By EFF and PI, we can assume that $\sigma_{i}=i$ for all $i \in N$. From now on, we assume that this is the case for all $\theta \in \Theta$. Since $\sigma$ is fixed, for all $\theta$, we denote the transfer specified by $\varphi^{S B}$ by $t^{S B}(\theta)$.

The proof is by induction. We first show that if $\theta=\left(\theta_{n}^{\prime}, \ldots, \theta_{n}^{\prime}\right)$, then $u_{i}\left(\sigma_{i}, t_{i} ; \theta_{i}\right)=u_{i}\left(\sigma_{i}, t_{i}^{S B}(\theta) ; \theta_{i}\right)$ for all $i \in N$ and all $(\sigma, t) \in \varphi(\theta)$. Assuming that this is true for all $\tilde{\theta} \leq \theta, \tilde{\theta} \in \Theta$, we then show that it is also true for $\theta$. Given PI, the essential single-valuedness of $\varphi^{S B}$, and the tree structure of the set $\Theta$, this proves the theorem. ${ }^{4}$

Initial Step: Let $\theta=\left(\theta_{n}^{\prime}, \ldots, \theta_{n}^{\prime}\right)$. By ETE and BB, $u_{i}\left(\sigma_{i}, t_{i} ; \theta_{i}\right)=$ $-(n-1) \theta_{n}^{\prime} / 2$ for all $i \in N$. Since $\varphi^{S B}$ satisfies ETE and BB, we have $u_{i}\left(\sigma_{i}, t_{i} ; \theta_{i}\right)=u_{i}\left(\sigma_{i}, t_{i}^{S B}(\theta) ; \theta_{i}\right)$ for all $i \in N$.

Induction Step: Suppose that for all $\tilde{\theta} \leq \theta=\left(\theta_{1}, \ldots, \theta_{n}\right), \tilde{\theta} \in \Theta$, and all $(\sigma, \tilde{t}) \in \varphi(\tilde{\theta}), u_{i}\left(\sigma_{i}, \tilde{t}_{i} ; \tilde{\theta}_{i}\right)=u_{i}\left(\sigma_{i}, t_{i}^{S B}(\tilde{\theta}) ; \tilde{\theta}_{i}\right)$ for all $i \in N$.

Step 1: Starting with $i=1$, we apply the following argument successively to $i=2, \ldots, n-1$.

Let $k \in N$ be the largest agent such that $\theta_{k}=\theta_{i}$. If $k=n$, proceed to Step 2.5 If $k<n$, let $\theta^{i}$ be a $k$-variant of $\theta$ such that $\theta_{k}^{i}=\theta_{k+1}$ and $\theta_{j}^{i}=\theta_{j}$ for all $j \neq k$. Observe that $\theta^{i} \in \Theta$ and $\theta^{i} \leq \theta$. Let $\left(\sigma, t^{i}\right) \in \varphi\left(\theta^{i}\right)$. By U-INV, $u_{k}\left(\sigma_{k}, t_{k} ; \theta_{k}\right)=u_{k}\left(\sigma_{k}, t_{k}^{i} ; \theta_{k}^{i}\right)$ and by the induction hypothesis, $u_{k}\left(\sigma_{k}, t_{k}^{i} ; \theta_{k}^{i}\right)=u_{k}\left(\sigma_{k}, t_{k}^{S B}\left(\theta^{i}\right) ; \theta_{k}^{i}\right)$. Since $\varphi^{S B}$

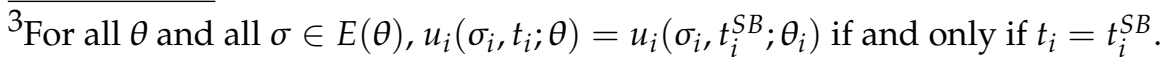

${ }^{4}$ See Figure 1 for an illustration of the tree structure. Note that every path emanating from a node $\theta$ ends at $\left(\theta_{n}^{\prime}, \cdots, \theta_{n}^{\prime}\right)$.

${ }^{5}$ Since $\theta_{1} \geq \cdots \geq \theta_{n}$, if $k=n$ for some $i$, then $k=n$ for all subsequent $i$. Hence Step 1 ends the first time $k=n$ for some $i$.
} 
satisfies U-INV, $u_{k}\left(\sigma_{k}, t_{k}^{S B}(\theta) ; \theta_{k}\right)=u_{k}\left(\sigma_{k}, t_{k}^{S B}\left(\theta^{i}\right) ; \theta_{k}^{i}\right)$. Therefore, $u_{k}\left(\sigma_{k}, t_{k} ; \theta_{k}\right)=u_{k}\left(\sigma_{k}, t_{k}^{S B}(\theta) ; \theta_{k}\right)$. Applying ETE to $\varphi$ and $\varphi^{S B}$, we have $u_{i}\left(\sigma_{i}, t_{i} ; \theta_{i}\right)=u_{i}\left(\sigma_{i}, t_{i}^{S B}(\theta) ; \theta_{i}\right)$.

Step 2: Let $N_{n}=\left\{j \in N \mid \theta_{j}=\theta_{n}\right\}$ be the set of agents whose waiting cost is $\theta_{n}$. By ETE, for all $j \in N_{n}, u_{j}\left(\sigma_{j}, t_{j} ; \theta_{j}\right)=u_{n}\left(\sigma_{n}, t_{n} ; \theta_{n}\right)$. Since $u_{j}\left(\sigma_{j}, t_{j} ; \theta_{j}\right)=u_{j}\left(\sigma_{j}, t_{j}^{S B}(\theta) ; \theta_{j}\right)$ for all $j \in N \backslash N_{n}$ by Step 1 , applying BB to $\varphi$ and $\varphi^{S B}$, we conclude that for all $j \in N_{n}, u_{j}\left(\sigma_{j}, t_{j} ; \theta_{j}\right)=$ $u_{j}\left(\sigma_{j}, t_{j}^{S B}(\theta) ; \theta_{j}\right)$.

We have thus shown for all $(\sigma, t) \in \varphi(\theta), u_{i}\left(\sigma_{i}, t_{i} ; \theta_{i}\right)=$ $u_{i}\left(\sigma_{i}, t_{i}^{S B}(\theta) ; \theta_{i}\right)$ for all $i \in N$. This establishes the induction step and proves the theorem.

Remark 3.2. Similar to upward-invariance (U-INV), we can define downward-invariance (D-INV) requiring that a unilateral decrease in an agent's waiting cost leave his transfer unchanged if the queue is unchanged. Starting from $\left(\theta_{1}^{\prime}, \ldots, \theta_{1}^{\prime}\right)$ and decreasing the waiting costs of agents sequentially to $\left(\theta_{1}^{\prime}, \ldots, \theta_{n}^{\prime}\right)$, we will obtain a similar (but different) tree. The proof technique of Theorem 3.1 can be easily adapted to show that a rule satisfies EFF, BB, ETE, PI and D-INV if and only if it is the SB rule.

Remark 3.3. Kayi and Ramaekers [5] show (Theorem 3(1), pp. 227) that only subcorrespondences of the SB rule satisfy EFF, BB, ETE and WSP. In a subcorrespondence of the SB rule, some efficient queues may not be chosen at a profile. In order to characterize the SB rule (Theorem 3(3), pp. 227), they replace ETE by symmetry which says that if an allocation is chosen at a profile $\theta$ where $i$ and $j$ have the same waiting cost, then the allocation where $j$ receives $i$ 's allocation and vice versa must also be chosen by the allocation rule. ${ }^{6}$

Definition. A rule $\varphi$ satisfies symmetry (SYM) if for all $\theta$, all $(\sigma, t) \in$ $\varphi(\theta)$ and all $i, j \in N$ such that $i \neq j$ and that $\theta_{i}=\theta_{j}$, if $\left(\sigma^{\prime}, t^{\prime}\right) \in$ $Z(\theta)$ is such that $\left(\sigma_{i}^{\prime}, t_{i}^{\prime}\right)=\left(\sigma_{j}, t_{j}\right),\left(\sigma_{i}^{\prime}, t_{j}^{\prime}\right)=\left(\sigma_{i}, t_{i}\right)$, and for all $\ell \in$ $N \backslash\{i, j\},\left(\sigma_{\ell}^{\prime}, t_{\ell}^{\prime}\right)=\left(\sigma_{\ell}, t_{\ell}\right)$, then $\left(\sigma^{\prime}, t^{\prime}\right) \in \varphi(\theta)$.

However, Theorem 3(3) is flawed since we can find a family of extended symmetrically balanced VCG rules parametrized by $\alpha$, $\varphi^{E S B \alpha}$, satisfying EFF, BB, SYM and WSP, defined as follows.

Let $\alpha \in \mathbb{R}_{+}$be given. For each $(\sigma, t) \in \varphi^{S B}(\theta)$, and each pair $\{i, j\} \subseteq N$, let $\left(\sigma^{i j}, t^{i j}\right)$ be such that $\sigma^{i j}=\sigma, t_{i}^{i j}=t_{i}+\alpha, t_{j}^{i j}=t_{j}-\alpha$,

\footnotetext{
${ }^{6}$ In their corrigendum, Kayi and Ramaekers [6] use equal treatment of equals in welfare along with symmetry, Pareto efficiency and strategy-proofness to characterize the $\mathrm{SB}$ rule.
} 
and $\sigma^{j i}=\sigma, t_{i}^{j i}=t_{i}-\alpha, t_{j}^{j i}=t_{j}+\alpha$; for all $k \notin\{i, j\}, t_{k}^{i, j}=t_{k}^{j, i}=t_{k}$. For all $\theta$, let $\varphi^{E S B \alpha}(\theta)$ be the set of allocations including all $(\sigma, t) \in$ $\varphi^{S B}(\theta)$, together with all $\left(\sigma^{i j}, t^{i j}\right)$ and $\left(\sigma^{j i}, t^{j i}\right)$.

It is not difficult to check that the family of extended symmetrically balanced VCG rules satisfies all the axioms of Kayi and Ramaekers [5], EFF, BB, SYM and WSP. Observe that this rule does not satisfy ETE if $\alpha \neq 0$.

Remark 3.4. There is no logical relationship between PI and SYM. However, our proof will go through if SYM is assumed instead of PI.

Remark 3.5. To check the independence of our axioms, we show that dropping one of EFF, BB, ETE, PI and U-INV gives rules different from the symmetrically balanced VCG rule.

(1) Let $N=\{1,2,3\}$. Consider the mechanism $(\sigma, t)$ where $\sigma$ is chosen according to the reverse-efficient order. (That is, $\theta_{i}>$ $\theta_{j}$ implies $\sigma_{i}>\sigma_{j}$.) The transfers for the profile $\theta_{1} \geq \theta_{2} \geq \theta_{3}$ and $\sigma_{1}>\sigma_{2}>\sigma_{3}$ are given by $t_{1}(\theta)=\theta_{2}=-t_{3}(\theta), t_{2}(\theta)=$ 0 . This mechanism satisfies all axioms except EFF.

(2) Let $\mathbf{e}=(1, \ldots, 1)$. The mechanism $\left(\sigma^{S B}, t^{S B}+\mathbf{e}\right)$ satisfies all axioms other than BB.

(3) Let $\mathbf{c}=\left(c_{1}, \ldots, c_{n}\right)$ where $c_{i} \neq c_{j}, i \neq j$ and $\sum_{i=1}^{n} c_{i}=0$. The mechanism $\left(\sigma^{S B}, t^{S B}+\mathbf{c}\right)$ satisfies all axioms other than ETE.

(4) Take a linear order $\succ$ on the set of queues $\Sigma(N)$. Consider the following mechanism $(\sigma, t)$. At any profile where there is more than efficient queue, the mechanism selects one queue using the order $\succ$. For this queue, transfers are chosen according to the SB-rule. This mechanism satisfies all axioms except PI.

(5) The minimum and maximum transfer rules (Maniquet [7], Chun [1]) satisfy all axioms except U-INV.

Remark 3.6. Our characterization will obviously go through if we replace U-INV by SP since U-INV is a weaker requirement. A natural question then is whether one of the other axioms (EFF, BB, ETE, PI) can be dropped. It is easy to show the necessity of BB, ETE and PI as dropping any one of them gives mechanisms which are not the SB rule. However, the necessity of EFF is not clear. Hashimoto and Saitoh [4] show that EFF is implied by SP and anonymity in welfare. The latter is stronger than ETE; hence, there is a gap which remains to be filled. 


\section{REFERENCES}

[1] Chun, Y., 2006a. A pessimistic approach to the queueing problem. Mathematical Social Sciences 51, 171-181.

[2] Chun, Y., 2006b. No-envy in queueing problems. Economic Theory 29, 151-162.

[3] Dolan, R. J., 1978. Incentive mechanisms for priority queuing problems. The Bell Journal of Economics 9, 421-436.

[4] Hashimoto, K. and Saitoh, H., 2012. Strategy-proof and anonymous rule in queueing problems: a relationship between equity and efficiency. Social Choice and Welfare 38, 3, 473-480.

[5] Kayi, C. and Ramaekers, E., 2010. Characterizations of Pareto-efficient, fair, and strategy-proof allocation rules in queueing problems. Games and Economic Behavior 68, 220-232.

[6] Kayi, C. and Ramekers, E., 2015. Corrigendum to "Characterizations of Pareto-efficient, fair, and strategy-proof allocation rules in queueing problems" [Games Econ. Behav. 68 (1) (2010) 220-232] Games and Economic Behavior, in press.

[7] Maniquet, F., 2003. A characterization of the Shapley value in queueing problems. Journal of Economic Theory 109, 90-103.

[8] Mitra, M., 2001. Mechanism design in queueing problems. Economic Theory $17,277-305$.

[9] Mitra, M. and Mutuswami, S., 2011. Group strategyproofness in queueing models. Games and Economic Behavior, 72, 242-254.

[10] Suijs, J., 1996. On incentive compatibility and budget balancedness in public decision making. Economic Design 2, 193-209. 


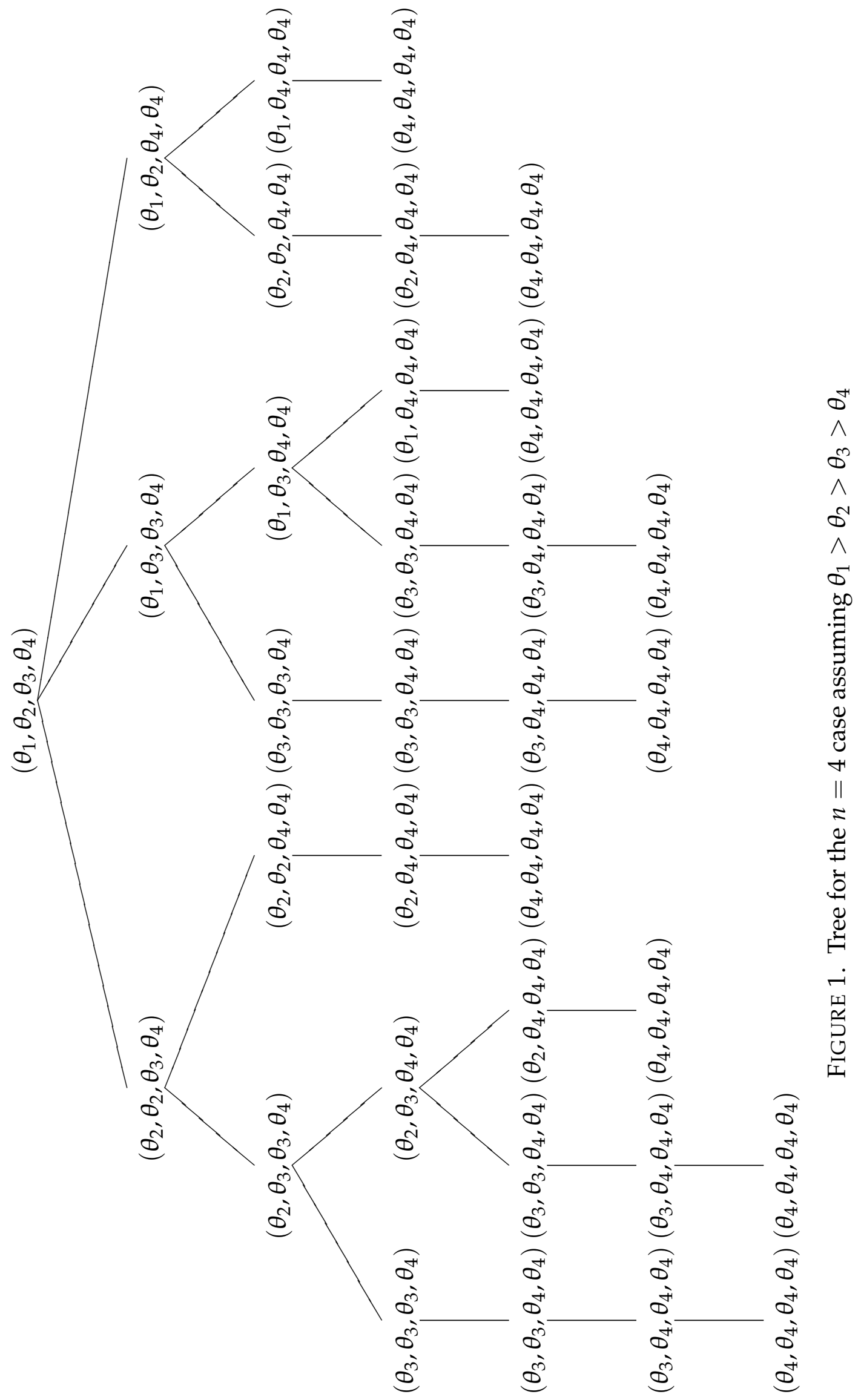

